\title{
Nanohistology of the Infectious Synapse: Correlated Fluorescent and Serial Ion Ablation-Scanning Electron Microscopy Reveals T Cell Collusion in HIV-1 Dissemination.
}

\author{
K. Olszens*, H. J. Yu**, N. Avishai***, Z. Wang**** A. Avishai***, A.H. Heuer*** and D. \\ McDonald* \\ *Departments of Molecular Biology \& Microbiology, ***Materials Science \& Engineering and \\ ****Biomedical Engineering, Case Western Reserve University, Cleveland, $\mathrm{OH}$. \\ **Drug Resistance Program, National Cancer Institute, Frederick, MD.
}

Pathogen activated dendritic cells (DCs) bind and concentrate HIV into a unique, surface connected storage compartment from which the HIV can emerge to infect CD4 T cells during natural immune contacts. This transfer of intact virions, referred to as trans-infection, substantially increases HIV replication in vitro and is thought to contribute to viral dissemination in vivo. We have previously shown that the virus compartment is recruited to the cellular interface between interacting DCs and T cells, and that individual virions traffic out of the pocket-like compartment to infect the $\mathrm{T}$ cell at the site of contact. Trans-infection is accompanied by recruitment of HIV entry receptors on the $\mathrm{T}$ cell, forming an "infectious synapse" that facilitates productive infection of the $\mathrm{T}$ cell [1,2]. To better understand the ultrastructure of the infectious synapse, we developed a correlative technique to visualize cells using fluorescent microscopy combined with serial ion ablation/scanning electron microscopy (SIA-SEM). SIA-SEM combines focused ion-beam milling with SEM to generate serial section images that can be rendered into 3D reconstructions, giving rise to whole-cell volumetric image datasets with sub-micron resolution. SIA-SEM has been used extensively in materials science [3], and recently has been employed to visualize the 3D organization of molecules and organelles within cells and tissues [4-5].

Our fluorescent microscopic analysis suggested that $\mathrm{T}$ cells extend membranous projections into the HIV storage pocket, and the presence of CD4 on these projections allows virions to transit the infectious synapse. SIA-SEM revealed that HIV is stored in highly compacted, invaginated membrane folds within the DCs that remain open to the extracellular milleau. Interacting $\mathrm{T}$ cells formed extensive contacts with the DCs, at times completely enveloped by DC membrane veils. Within the context of the synaptic structure, $T$ cells were observed to extend long-range surface protrusions toward the sequestered HIV, at times reaching thin "nanotubes" directly into the HIV pocket. Our data suggest that $\mathrm{T}$ cells play an active role in trans-infection by extending surface protrusions into the HIV pocket and fishing the virus out using CD4 as the "hook."

Correlated fluorescent microscopy and EM tomography provides a powerful new approach to visualizing the complex molecular interplay within cells and tissues. With careful sample preparation and image registration, resolution of fluorescently identified structures can be pushed into the nanometer realm.

\section{References}

1. D. McDonald et al., Science (2003) 300: 1295-7.

2. H.J. Yu et al., PLoS Pathogens (2008) 4: e1000134.

3. Yeoh et al., J. Vac. Sci. Technol. B (2007) 25(3): 922-925

4. Knott et al., The Journal of Neuroscience (2008) 28:2959-64.

5. J.A.W. Heymann, et al., J. Structural Bio. (2009) 166: 1-7.

6. This research was supported by NIAID grant AI087511-01A1 (DM) and the Case/UH Center for

AIDS Research (DM, KO). 

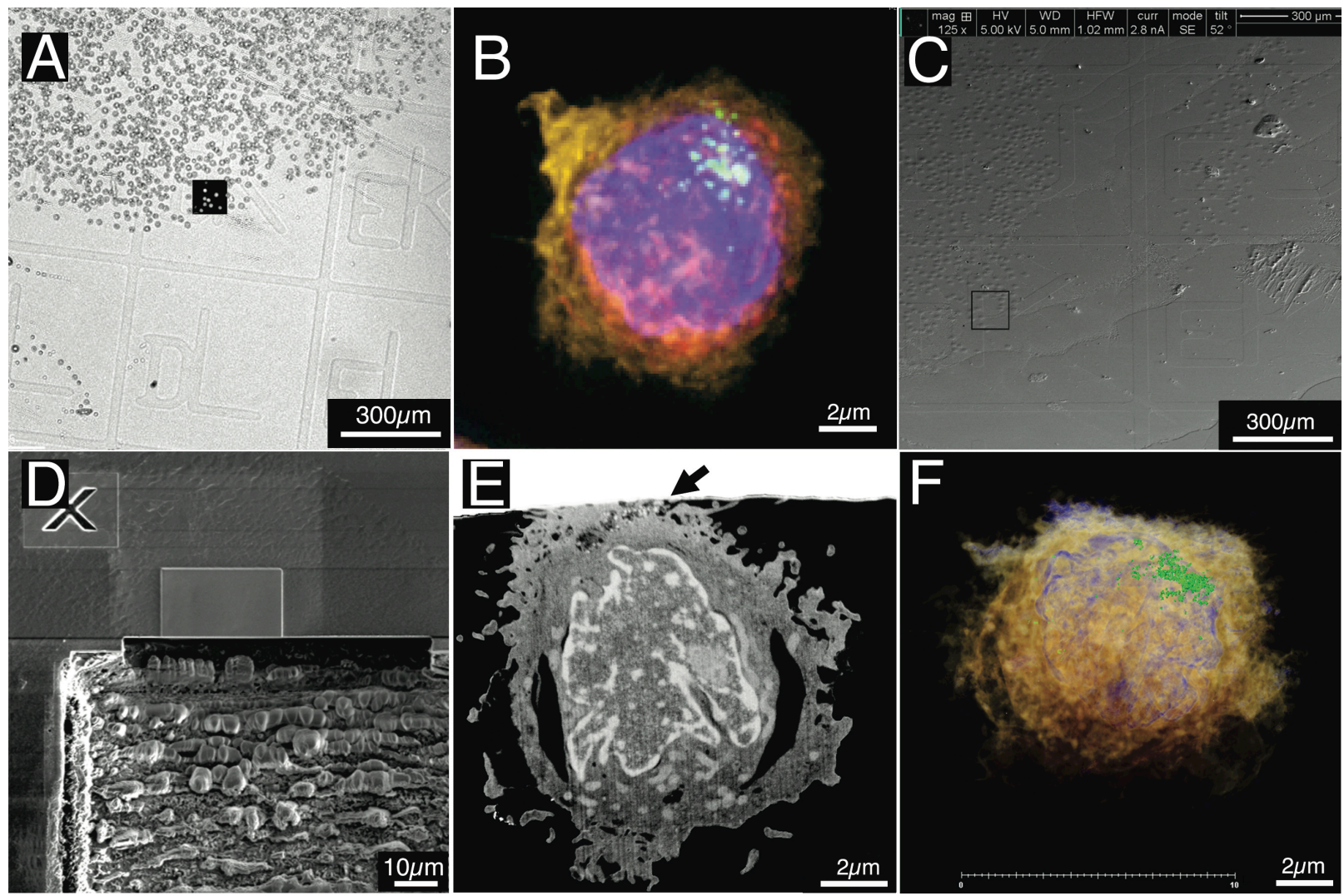

Figure 1. Correlated fluorescent and 3D reconstructed SIA-SEM images. A-B. DCs carrying GFP-HIV were fixed onto gridded coverslips and imaged with an inverted microscope at low and high magnification. C. Cells were embedded and prepared for FIB-SEM. Note the grid pattern is clearly visible, black box denotes relocated cells. D. DC was relocated, overlaid with a protective $\mathrm{Pt}$ pad and FIB trenched in front of the cell. E. SIA-SEM was performed on the exposed face. Note HIV particles in the upper portion of the cell (arrow). F. 3D reconstruction of SEM data. Colors (B, F): green, HIV; orange, cell membrane; blue, nucleus.
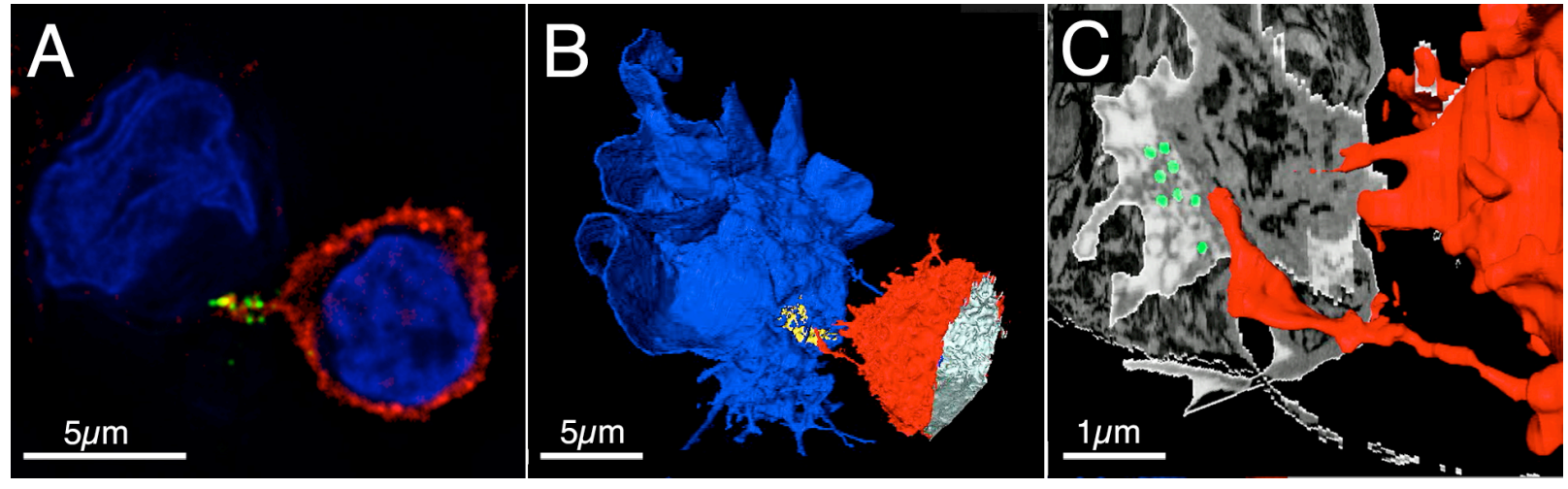

Figure 2. Correlated fluorescent and SIA-SEM imaging of the infectious synapse. A. Fluorescent micrograph of CD4 T cell (red) extending membranes into contact with HIV (green) within the DC. Blue, nuclei; the DC membrane stain is not shown here for clarity. B. Serial EM images were manually segmented to identify DC (blue), T cell (red), HIV (green) and the HIV containing pocket structure (yellow). C. Magnified view of T cell projection (red) shown with EM section, individual HIV particles are labeled green. 